J. Lake Sci. (湖泊科学), 2018, 30(5): 1187-1195

DOI 10. 18307/2018. 0502

(c) 2018 by Journal of Lake Sciences

\title{
老碳对水生态系统食物网的贡献
}

胡 恩 $^{1,2}$, 张振文 ${ }^{1}$, 王文科 ${ }^{2}$, 孙长顺 ${ }^{1}$, 苏雅玲 ${ }^{3}$, 刘正文 ${ }^{3,4,5}$

(1:陕西省环境科学研究院,西安 710069)

( 2 : 长安大学环境与工程学院, 西安 710069)

(3: 中国科学院南京地理与湖泊研究所,南京 210008)

(4:暨南大学生态学系与水生生物研究中心,广州 510632)

(5: 中国科学院大学中丹学院, 北京 100049)

摘 要: 传统理论认为, 支撑水生态系统食物网的碳主要来自于系统内部的初级生产者, 如藻类、大型水生植物等, 或者 来自陆源输人的新鲜且容易分解的有机质; 而部分生物或非生物生产的有机碳, 在冰川、冻土等环境中可停留数百年至 数千年 (定义为老碳), 由于储存环境稳定、物理化学性质顽固, 难以参与水生生态系统有机碳循环. 近年来这一观念不断 被挑战. 研究表明老碳能够参与水生态系统食物网碳循环: 可以被微生物分解利用; 被浮游动物、无脊椎动物直接或者间 接摄取、或沿着营养级传递至鱼类、水禽等高级消费者. 这意味着除了内部初级生产, 老碳是支撑水生态系统重要的碳源. 本文概述了当前老碳与水生态系统食物网关系研究最新进展和所取得的研究成果, 介绍放射性 ${ }^{14} \mathrm{C}$ 同位素技术在水生态 学领域的应用, 同时提出研究中存在的问题以及未来研究应关注的方向, 以期促进我国水生态系统碳循环研究的进一步 发展.

关键词: 水生态系统; 食物网; 老碳; ${ }^{14} \mathrm{C}$ 同位素技术

\section{Review on contribution of ancient organic carbon to aquatic food webs}

HU En ${ }^{1,2}$, ZHANG Zhenwen ${ }^{1}$, WANG Wenke ${ }^{2}$, SUN Changshun ${ }^{1}$, SU Yaling ${ }^{3}$ \& LIU Zhengwen ${ }^{3,4,5}$

(1: Shaanxi Provincial Academy of Environmental Science, Xi'an 710069, P.R.China)

(2: School of Environmental Science and Engineering, Chang'an University, Xi'an 710069, P.R.China)

(3: Nanjing Institute of Geography and Limnology, Chinese Academy of Sciences, Nanjing 210008, P.R.China)

(4: Institute of Hydrobiology, Jinan University, Guangzhou 510632, P.R.China)

(5: Sino-Danish Centre for Education and Research / Sino-Danish College of University of Chinese Academy of Sciences, Beijing 100049, P.R.China)

Abstract: Traditionally, aquatic ecosystem consumers are considered to mainly feed on primary production derived organic carbon (OC) from contemporary photosynthesis (e.g. algae, macrophytes) or on fresh terrestrial OC from watershed that is less refractory than previously thought. Due to structure stability and stubborn physicochemical feature, some biotical or abiotic OC stored in glacier and permafrost systems for hundreds to thousands years (defined as ancient or highly aged OC) may be hardly incorporated into biogeochemistry process in aquatic ecosystems. Recent studies, however, have increasingly revealed that ancient OC may be an important resource for microbes, zooplankton, invertebrates, and higher trophic consumers such as fish and waterfowl and significantly subsidizes food webs of aquatic ecosystems. The ancient OC of aquatic consumers indicates that a shortage of modern primary production may not necessarily limit or constrain the food supply to consumers in aquatic ecosystems. In this paper, we have summarized the research progresses on the relationship between terrestrial ancient $O C$ and aquatic food webs worldwide, introduced the application of ${ }^{14} \mathrm{C}$ technology in aquatic ecology, and pointed out the problems existing in the studies and the direction in the future research. This study will promote the research of carbon cycle of aquatic ecosystems in China.

Keywords: Aquatic ecosystems; food webs; ancient organic carbon; radiocarbon isotope technology

* 国家自然科学基金项目(31670461) 和陕西省博士后基金项目(2017BSHQYXMZZ10)联合资助. 2017-11-20 收稿; 2018-01-07 收修改稿. 胡恩(1983 ), 男,博士; E-mail: huen777@ 163.com. 
传统观念中, 水生态系统中异养生物的碳主要来自于系统内部的初级生产者 (内源碳), 如浮游藻类、大 型水生植被等; 或者来自陆源有机碳 (外源碳 $)^{[1-3]}$. 外源有机碳中包含部分储存时间数百年至数千年的有机 碳(定义为“ancient organic carbon’或者“highly aged organic carbon’), 本文称之为老碳. 当前学术界的主流 观点认为: 高龄有机碳生物地球化学特性比较顽固, 不易于分解利用, 从而难以参与水生态系统有机碳循 环 ${ }^{[4-6]}$. 如今, 这一观点似乎并非那么牢靠 ${ }^{[7-10]}$. 研究表明, 老碳难以参与水生态系统有机碳循环, 并非自身 物理化学性质所致, 而是和其所处环境的稳定性有关 ${ }^{[11]}$. 长期以来, 冰冻圈、陆表土壤等系统环境稳定, 在 一定程度上对存储其中的有机碳起着庇护作用. 而随着外界环境条件变化 (气候变化和人类活动等), 此类 “庇护所”稳定性变差, 老碳便经由地表径流、地下水等途径进人水生态系统 ${ }^{[12-18]}$.

长期以来, 由于技术手段局限, 老碳在水生态系统食物网的功能与所扮演的角色并未引起学者们足够 重视. 随着放射性 ${ }^{14} \mathrm{C}$ 同位素技术方法的应用, 相关研究得以开展, 尤其是在全球变化响应迅速的高纬度、高 海拔地区, 如北极、青藏高原. 在上述地区研究发现, 源自冰川、冻土的老碳生物可利用性高, 能够广泛参与 水生态系统食物网有机碳循环: 如被微生物分解利用 ${ }^{[14-15]}$; 被浮游动物、无脊椎动物直接或者间接摄 食 ${ }^{[19-23]}$, 或传递至鱼类 ${ }^{[19-20]}$ 、水禽 ${ }^{[24]}$ 等高级消费者甚至陆地消费者 ${ }^{[21]}$. 在非高纬度和高海拔地区的水生态 系统中, 来自流域或者深层土壤中的老碳同样可以为水生态系统提供碳源 ${ }^{[25-31]}$. 由此可见, 老碳是水生态系 统消费者的重要有机碳来源之一( 图 1 和表 1 ).

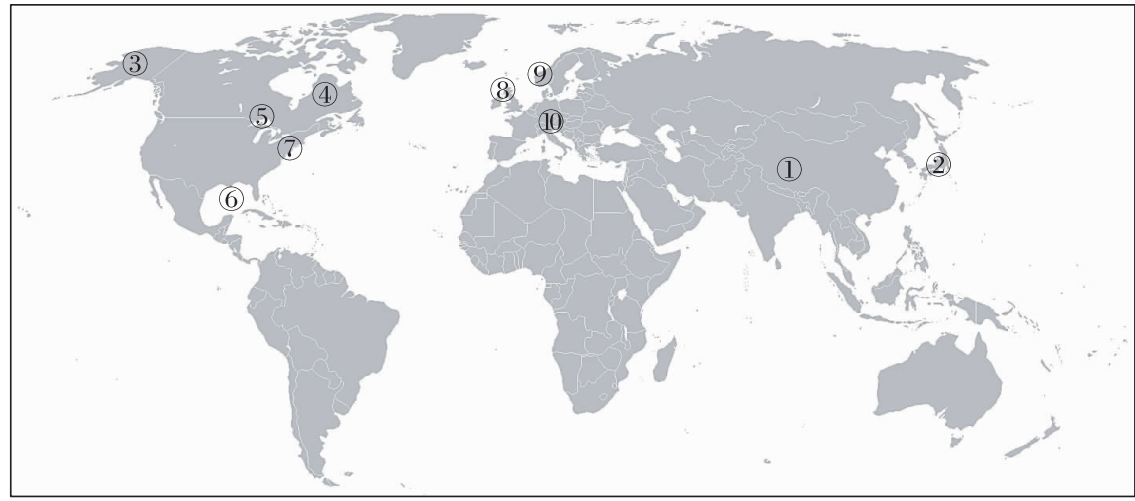

图 1 全球水生态系统食物网与老碳关系研究

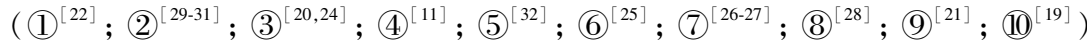

Fig.1 Studies of relationship between ancient organic carbon and aquatic food webs worldwide

表 1 水生态系统消费者 ${ }^{14} \mathrm{C}$ 特征及来源

Tab.1 The ${ }^{14} \mathrm{C}$ signature and its origin of consumers in aquatic food webs worldwide

\begin{tabular}{cccccc}
\hline 地点 & 研究系统 & 消费者类型 & $\Delta^{14} \mathrm{C}$ 比值/\%o & 系统老碳来源 & 文献 \\
\hline Alaska & 海洋 & 鱼类、水禽 & $-150 \sim 209$ & 溶解有机碳 & {$[24]$} \\
USA & 海洋 & 微生物 & $-61 \sim 132$ & 溶解有机碳 & {$[25]$} \\
Norway & 河流 & 底栖动物 & $-334 \sim-41.4$ & 颗粒有机碳 & {$[21]$} \\
Alaska & 河流 & 底栖动物、鱼类 & $-172 \sim 22$ & 溶解有机碳 & {$[20]$} \\
USA & 河流 & 浮游动物 & -236 & 颗粒有机碳 & {$[27]$} \\
USA & 河流 & 底栖动物 & $-75 ~ 34$ & 有机碳、碳酸盐 & {$[23]$} \\
Canada & 湖泊、河流 & 微生物 & $-172 ~ 94$ & 有机碳 & {$[11]$} \\
Japan & 湖泊、河流 & 底栖动物、鱼类 & $-178 ~ 68$ & 碳酸盐 & {$[29-31]$} \\
USA & 湖泊 & 浮游动物 & $57 \sim 62$ & 碳酸盐 & {$[32]$} \\
Northern Ireland & 湖泊 & 浮游动物、鱼类 & $-108.1 \sim-52.7$ & 有机碳、碳酸盐 & {$[28]$} \\
Germany & 湖泊 & 底栖动物、鱼类 & $-539.3 \sim-8.3$ & 碳酸盐 & {$[19]$} \\
China & 湖泊 & 浮游动物 & -45.3 & 有机碳 & {$[22]$} \\
\hline
\end{tabular}


老碳的水生态学功能研究起步较晚, 外加 ${ }^{14} \mathrm{C}$ 测试价格不菲, 已有研究成果不多且绝大多数集中在海洋 以及河流生态系统 ${ }^{[33-34]}$, 定量揭示老碳和水生态系统食物网关系的研究鲜有耳闻 ${ }^{[20,22,27,31]}$. 基于此, 本文根 据现有文献资料, 总结全球水生态系统食物网老碳研究进展与所取得的成果, 介绍放射性 ${ }^{14} \mathrm{C}$ 同位素技术在 水生态学领域的应用, 指出研究中存在的问题以及未来研究应关注的方向, 以期为我国水生态系统食物网 碳循环研究的发展提供借鉴和参考。

\section{1 老碳形态及食物网传递途径}

水体中老碳主要以两种形态存在: 颗粒有机碳 (particulate organic carbon, POC) 与溶解有机碳 ( dissolved organic carbon, DOC). 二者都可以为食物网消费者提供碳源, 但参与食物网碳循环的方式和途径 有所不同, 对食物网碳源贡献额度也存在差别. POC 主要是由植物碎屑、异养生物和非生命有机物组成的混 合物, 而 DOC 是由腐殖酸组成的复杂混合物. 一般情形下, POC 可以被消费者直接摄取、同化吸收 ${ }^{[35-36]}$; 而 DOC 则需要通过微生物利用, 然后转化为有机碎屑再被消费者所摄食 ${ }^{[37-39]}$. 现有研究表明: 大多数情形下, 老碳以 DOC 的形态参与碳循环, 经由微生物分解利用, 沿着食物网传递 (图 2). 例如, 在北太平洋, 海水中细 菌优先选择性利用 DOC 老碳 ${ }^{[25]}$; 在温带湖泊和河流, POC 老碳则来自于微生物所转化的 DOC, 再传递至消 费者 ${ }^{[11]}$; 阿拉斯加冰川河流, 吸附有 DOC 老碳的生物膜优先被无脊椎动物所摄食, 随后传递至鱼类等高级 消费者 ${ }^{[20]}$. 相比 POC, 老碳以 DOC 的形态似乎更容易进人食物网, 尤其是初级生产力低的寡营养和富含腐 殖质的小型水体中, 这主要归因于 DOC 的易降解性和较高的生物可利用性 ${ }^{[14]}$. 食物质量往往是决定消费者 选择摄取哪种有机碳的重要影响因素. 在冰冻圈, 水体中 POC 主要源于冰川侵蚀流域下伏基岩产生的有机 碎屑, 其营养水平低、食物质量差, 往往不被消费者所青睐 ${ }^{[40]}$. 当然情况并非绝对, 美国 Hudson 河流, 浮游动 物食物来源主要为 POC 老碳, 尽管新鲜生产的 DOC 占据有机碳库的主导地位 ${ }^{[27]}$. 除了有机碳 (POC 和 DOC), 流域碳酸盐所带来的水体碳库效应同样会使消费者获取老碳特征 (图 2), 该部分内容将在第 5 节中 详细阐述.

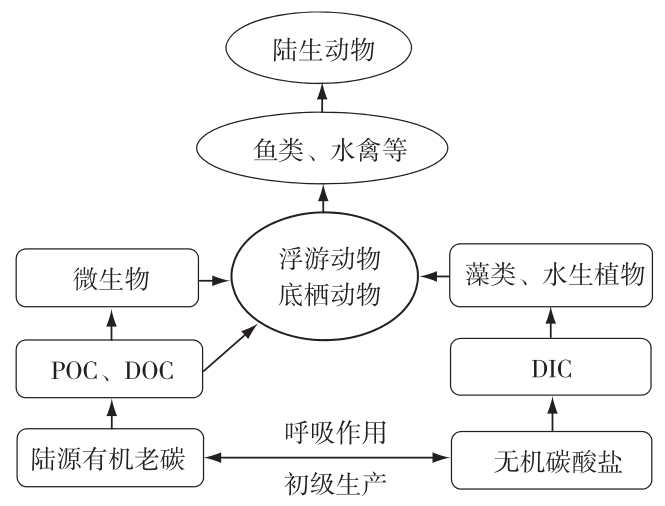

图 2 水生态系统食物网老碳特征来源及传递途径 (POC: 颗粒有机碳; DOC: 溶解有机碳; DIC: 溶解无机碳)

Fig.2 The sources and path ways of ancient carbon signature in aquatic food webs

(POC: particulate organic carbon; DOC: dissolved organic carbon; DIC: dissolved inorganic carbon)

\section{2 水生态系统老碳来源}

全球范围内, 老碳主要来源于高纬度、高海拔冰冻圈的冰川、冻土, 沉积岩、陆表深层土壤以及海洋、湖 泊沉积物等介质环境中, 在农业生产、城市化进程等人类活动以及气候变化、极端降水等自然事件等背景下 进人水生态系统. 


\section{1 冰川}

冰冻圈 ( cryosphere) 是地球气候系统五大圈层之一, 是地球表层和气候系统的重要组成部分, 主要由地 球陆地和海洋表面及以下的积雪、冰川、冻土以及海冰、湖冰与河冰等组成 ${ }^{[41]}$. 气候变暖驱动下, 冰冻圈逐 渐成为有机碳最为活跃的场所, 在全球碳循环过程中扮演越来越重要的角色 ${ }^{[42]}$. 据科学估算, 仅北极圈存 储的有机碳占全球总量的 $25 \% \sim 33 \%{ }^{[43-44]}$. 全球冰川总面积达 $14.5 \times 10^{6} \mathrm{~km}^{2}$, 约占地球陆表 $11 \%$ 的面积, 主 要分布于两极地区以及中、低纬度高海拔地区, 如青藏高原 ${ }^{[45-46]}$. 冰川储存的老碳主要源于冰川前进时所覆 盖的陆生植物或者来自于大气沉降的有机碳 ${ }^{[4]}$. 研究表明, 格陵兰岛冰架、阿拉斯加冰原以及青藏高原山 岳冰川每年可输出相当数量老碳, 伴随着冰川径流进人海洋、河流以及湖泊 ${ }^{[42]}$.

\section{2 土壤}

地球陆表土壤蕴含有机碳数量庞大, 一般土壤层深度越深, 埋藏的有机碳年代越老. 气候变化驱动温度 和水文特征变化是控制土壤有机碳通量的重要自然因素 ${ }^{[47-49]}$. 土壤有机碳动态过程对气候变化响应十分敏 感,全球变暖背景下, $1988-2000$ 年间, 英国的泥炭沼泽输出的溶解有机碳增加了约 $65 \%^{[50]}$. 除了自然因 素, 人类活动扰动显著改变着地球陆表形态, 使得土层中的老碳经由人类活动 (农耕生产、城市化进程) 或者 自然事件 (极端降水) 输送至水生态系统, 参与有机碳循环 ${ }^{[51]}$.

相比其他土壤类型, 高纬度、高海拔的多年冻土对气候变化响应更为迅猛, 有机碳输出量增长速率尤为 显著. 冻土老碳数量庞大, 主要源于间冰期生长的陆生植被, 腐烂死亡后经历埋藏作用存储在冻土层中. 据 估算, 约有 $1672 \mathrm{Pg}$ 的有机碳埋藏在北半球极地冻土, 其中包含数量庞大的老碳 ${ }^{[22]}$. 至本世纪末, 西伯利亚 地区年平均气温会比现在提升约 $2^{\circ} \mathrm{C}$, 冻土层将迅速退化, 输出的溶解性有机碳数量将增加 $670 \%{ }^{[47]}$. 此外, 冰川消融导致地表径流增加, 伴随着热融、流水侵蚀等外力作用, 冻土层将释放更多的有机碳, 由地下水或 者地表径流进人水体 ${ }^{[53]}$, 可以预见, 冻土有机碳将显著改变全球碳收支平衡以及各生态圈层的有机碳循环 过程 ${ }^{[54-55]}$.

\section{3 沉积物}

据估算, 约 $15000000 \mathrm{Pg}$ 有机碳存储于沉积岩中, 沉积岩有机质年代老 (>50000 a), 且以难溶性的大分 子、长链有机质为主 ${ }^{[33,56]}$. 一直以来, 此类有机质被贴上稳定性高、物理化学性质顽固的标签, 加上沉积环境 稳定 (地表下), 难以和地表大气圈、水圈等相对活泼的圈层相互作用. 近年来, 有研究猜测沉积岩中的有机 质或许可以被河流中的生物所利用, 但是仍然缺乏直接的证据 ${ }^{[34]}$. 海洋和湖泊, 接受来自流域和系统内部 形成的年代各异的有机质, 经过长期沉降, 转化为老碳存储于沉积物中. 在水体对流交换和再悬浮作用下, 表层沉积物释放老碳进人上层水体, 直接或者间接被水中消费者所摄取, 参与食物网碳循环. 因此, 沉积物 可能是水生态系统老碳另一潜在来源. 研究表明, 沉积物中的老碳不仅可以被浮游动物所摄取, 同样是底栖 动物重要的有机碳来源 ${ }^{[28]}$. 笔者利用放射性 ${ }^{14} \mathrm{C}$ 同位素方法对青藏高原边缘地区开展高山湖泊食物网调查 研究, 沉积物中的老碳是湖泊表层水体有机碳库的重要补充, 亦是底栖生物消费者有机碳的重要来源 (未发 表数据).

\section{3 放射性 ${ }^{14} \mathrm{C}$ 同位素及混合同位素模型在水域生态学的应用}

\section{1 放射性 ${ }^{14} \mathrm{C}$ 同位素}

稳定同位素技术 $\left({ }^{13} \mathrm{C} 、{ }^{15} \mathrm{~N} 、{ }^{2} \mathrm{H} 、{ }^{34} \mathrm{~S}\right.$ 等 $)$ 是揭示水生态系统物质传递、能量流动以及食物网营养关系的重 要手段和工具, ${ }^{13} \mathrm{C}$ 技术则被广泛应用于追踪食物网的碳流方向 ${ }^{[57]}$. 然而, ${ }^{13} \mathrm{C}$ 同位素有其应用的局限性: 首 先, 自然界大多数碳源的 $\delta^{13} \mathrm{C}$ 值皆分布在一个狭窄的范围 $(-32 \% 0 \sim-12 \% 0)$, 若消费者所利用的碳源 $\delta^{13} \mathrm{C}$ 比 值相近, ${ }^{13} \mathrm{C}$ 方法则无法区分碳源之间同位素特征重叠的问题 ${ }^{[58]}$; 其次, 有机质形成年代存在差异 (如老碳 和新鲜有机质), ${ }^{13} \mathrm{C}$ 方法无法识别碳源之间年代的差别; 最后, 浮游植物是水生态系统中重要的初级生产 者, 测定其 $\delta^{13} \mathrm{C}$ 困难, 尤其是贫营养水体中 ${ }^{[59]}$. 天然丰度放射性 ${ }^{14} \mathrm{C}$ 同位素技术很好地解决了上述难题. 放 射性 ${ }^{14} \mathrm{C}$ 同位素采用加速器质谱仪 (AMS) 测定, 相比稳定同位素技术, ${ }^{14} \mathrm{C}$ 拥有更为宽广的测试范围 $\left(-1000 \% 0 \sim 200 \%\right.$ ) , 从而解决了稳定同位素重叠而导致碳源难以区分的问题 ${ }^{[60-61]}$; 通过测定天然放射性碳 同位素并建立高精度年代标尺, 可以有效区分不同碳源之间的年代差异, 反映有机物在水体和流域内的时 
空变化 ${ }^{[32]}$. 此外可以通过测试水体 DIC 的 ${ }^{14} \mathrm{C}$ 比值指代浮游植物的 ${ }^{14} \mathrm{C}$ 比值, 从而解决浮游藻类同位素比值 难以测定的问题 ${ }^{[61]}$.

放射性碳同位素比值用 $\Delta^{14} \mathrm{C}$ 表示, 通过 AMS 测定消费者及其潜在碳源的 $\Delta^{14} \mathrm{C}$, 依照 ${ }^{14} \mathrm{C}$ 的半衰期 (5730 a) 即可计算消费者和碳源的具体年代, 从而推断消费者是否利用了老碳. 此外再结合混合同位素模 型, 便能估算老碳对消费者的贡献额度. 可以说, ${ }^{14} \mathrm{C}$ 同位素技术方法能够胜任 ${ }^{13} \mathrm{C}$ 可以解决或者难以解决的 大多数问题,使得其在水域生态学等方面的应用前景极为广阔. $\Delta^{14} \mathrm{C}$ 计算公式为:

$$
\begin{gathered}
\Delta^{14} \mathrm{C}(\%)=\delta^{14} \mathrm{C}-2\left(\delta^{13} \mathrm{C}+25\right)\left(1+\delta^{13} \mathrm{C} / 1000\right) \\
\delta^{14} \mathrm{C}(\%)=\left(R_{\text {sample }} / R_{\text {standard }}-1\right) \times 10^{3}, R={ }^{14} \mathrm{C} /{ }^{12} \mathrm{C} \\
\delta^{13} \mathrm{C}(\%)=\left(R_{\text {sample }} / R_{\text {standard }}-1\right) \times 10^{3}, R={ }^{13} \mathrm{C} /{ }^{12} \mathrm{C}
\end{gathered}
$$

式中, ${ }^{14} \mathrm{C}$ 对应的国际标准为 1950 年草酸 (1950s oxalic acid) $;{ }^{13} \mathrm{C}$ 采用维也纳-PeeDee 箭石标准 (VPDB). 若 消费者的 $\Delta^{14} \mathrm{C}$ 测试结果为负值 $\left(\Delta^{14} \mathrm{C} \% 0<0\right)$, 表明其利用了 1950 年之前形成的有机碳; 若 $\Delta^{14} \mathrm{C}$ 为正值 $\left(\Delta^{14} \mathrm{C} \% 0>0\right)$, 则为 1950 年之后形成的有机碳. $\Delta^{14} \mathrm{C}$ 值越负, 表明消费者利用的有机碳年代越老. 一般情况 下, 样品的 $\Delta^{14} \mathrm{C}$ 比值所对应的放射性年代在 $200 \mathrm{a}$ 以下的, 可认定为现代碳; 对应年代介于 $200 \sim 50000 \mathrm{a} 之$ 间,则称之为老碳; 超过 $50000 \mathrm{a},{ }^{14} \mathrm{C}$ 方法便不能判断有机质的年代, 这也是 ${ }^{14} \mathrm{C}$ 方法应用的一个限制 ${ }^{[62]}$.

\section{2 混合同位素模型}

通过放射性 ${ }^{14} \mathrm{C}$ 同位素技术, 可以定性揭示消费者所利用碳源的年代特征. 为定量估算老碳对消费者的 贡献比例, 则需要借助于混合同位素模型. 目前应用较为广泛的有 IsoSource、Bayesian 等混合同位素模型. 此 类模型主要利用反复迭代的方法求解消费者食源贡献, 经验证已广泛应用于水域食物网的研究 ${ }^{[20,22,27]}$. 以 上模型是基于统计学方法建立的混合模型, 根据质量守恒方程, 计算出来的结果表征不同碳源对消费者贡 献的相对比例范围, 范围越小, 表明估算的结果越准确. 目前应用最广泛的同位素有 ${ }^{13} \mathrm{C} 、{ }^{15} \mathrm{~N} 、{ }^{34} \mathrm{~S} 、{ }^{18} \mathrm{O} 、{ }^{2} \mathrm{H}$ 以 及近年来兴起的放射性 ${ }^{14} \mathrm{C}$. 对于混合同位素模型而言, 原则上使用的同位素越多, 估算的结果也更为精确. 然而在实际应用过程中, 由于采集足够的样品量比较困难 (如贫营养水体中的浮游植物), 往往难以获取其 同位素比值. 因此, 应用混合同位素模型追溯食物网碳源时, 主要是基于 $\delta^{13} \mathrm{C}$ 和 $\Delta^{14} \mathrm{C}$ 两种同位素构建质量 守恒模型来求解多种碳源的贡献.

\section{4 老碳对水生态系统食物网贡献的控制因素}

尽管诸多研究已证实老碳等此类陆源有机质对水生态系统食物网碳源的贡献, 然而关于陆源有机质在 食物网中的功能和作用还是尚存争议 ${ }^{[63-65]}$. 一般而言, 在营养水平较高的水体中, 内源初级生产者丰富, 陆 源有机碳对食物网贡献额度一般比较小; 而在贫营养水体中, 由于系统内部初级生产受限, 陆源有机碳的贡 献对食物网往往具有决定性作用 ${ }^{[1-3]}$. 然而 Brett 等 $^{[36]}$ 和 Kelly 等 ${ }^{[64]}$ 发现, 在贫营养水体中,随着食物中陆源 有机碳比例的增加, 浮游动物的生产力和繁殖力都呈现显著的下降趋势, 从而认为陆源有机碳对浮游动物 群落生长有明显的抑制作用, 并将这一现象归因于: 1) 外源有机质包含腐殖质等有色物质, 减弱水体表层透 光率, 导致光合作用降低, 从而抑制浮游藻类等异养生物的光合作用以及初级生产力;2) 外源有机碎屑营养 (脂肪酸等) 价值低, 分解速率缓慢, 且含有大量的腐殖质、木质素以及纤维素等难降解的大分子物质, 属于 低质量食物, 消费者难以有效同化、吸收. 相比之下, 消费者更倾向于利用高质量的内源有机物 ${ }^{[36,66]}$. 总之, 陆源有机碳对水生态系统食物网是正向作用还是负面效应, 不可一概而论, 而是由水体的营养水平状态、陆 源有机质食物质量水平 ${ }^{[39]}$ 和消费者种类组成等因子共同决定 ${ }^{[67-70]}$, 可见, 陆源有机碳在水生态系统中的利 用效率与途径还需深人研究.

\section{5 碳库效应的影响}

前文提及,除了直接或间接摄取有机老碳, 由于水体碳库效应的影响 ( reservoir effects), 消费者也会呈现 老碳特征 $\left(\Delta^{14} \mathrm{C} \% 0_{0}<0\right)$. 海洋碳库效应是海洋表层水和深层水缓慢混合的结果. 表层水和大气 $\mathrm{CO}_{2}$ 交换速率 快, 并在短期内达到平衡, ${ }^{14} \mathrm{C}$ 浓度和大气 ${ }^{14} \mathrm{C}$ 含量一致, 然而海洋深层水与表层水的交换速率缓慢, 因此二 者的 ${ }^{14} \mathrm{C}$ 含量差异显著 (深层水 ${ }^{14} \mathrm{C}$ 浓度低于表层水). 全球海洋表层水 ${ }^{14} \mathrm{C}$ 含量由于受到上涌海水的稀释作 
用使得其表观年龄平均偏老 $400 \mathrm{a}$ 左右. 因此在进行海洋生物样品 ${ }^{14} \mathrm{C}$ 定年的时候,需要校正不同地区海洋 碳库效应的影响 ${ }^{[71-72]}$. 相比较海洋, 湖泊和河流则容易受到流域碳酸盐所带来的硬水效应的影响 ${ }^{[73-74]}$. 碳酸 盐 ${ }^{14} \mathrm{C}$ 含量极少或者完全衰减 ( $\Delta^{14} \mathrm{C}$ 接近- $1000 \%$ ), 在水体溶解后, 以 DIC 的形态被浮游藻类、沉水植物等 初级生产者通过光合作用固定, 导致初级生产者 ${ }^{14} \mathrm{C}$ 年代偏老, 消费者摄食初级生产者之后亦会呈现老碳特 征. 此外, 若水体中存在年代老的有机碳, 呼吸作用下被分解产生 $\mathrm{CO}_{2}$, 再溶解进人水体, 同样会导致碳库效 应. 因此在利用放射性 ${ }^{14} \mathrm{C}$ 方法开展水生态学方面等相关研究时, 要慎重考虑碳库效应对消费者老碳来源所 带来的影响. 现代水生态系统食物网 ${ }^{14} \mathrm{C}$ 研究对古生态学研究同样有参考和借鉴意义. 历史时期, 水体中曾 经摄取过老碳的消费者, 如甲壳类浮游动物、摇蚊等, 死亡后以化石的形态保存于沉积物中, 此后若对化石 进行 ${ }^{14} \mathrm{C}$ 定年, 由于老碳的影响, 会造成测试年代与实际年龄的不匹配或高估. 所以, 建议在沉积物化石年代 学研究时,考虑水体时代背景并去除区域环境因素的影响.

\section{6 未来研究展望}

近年来, 不断有研究证实老碳能够被水生态系统消费者摄取从而参与食物网的碳循环, 学者们也开始 重新评估和审视老碳和水生态系统碳循环之间的潜在关联, 并取得一些新的研究成果, 然而关于老碳在水 生态系统中扮演的角色、承担功能和作用仍有诸多疑惑需要解答. 本文综合前人研究成果, 尝试提出几个未 来值得关注的问题:

1) 消费者直接或者间接利用高龄有机碳 (ancient OC) 以及溶解无机碳 (ancient DIC) 产生的碳库效应均 可以带给消费者老碳特征. 而水体碳库效应一般有两种途径: 一是风化作用将流域碳酸盐带进水体; 二是呼 吸作用分解高龄有机碳, 释放出的 $\mathrm{CO}_{2}$ 溶解进人水体. 所以在存在碳库效应的水体中, 区分碳库效应的明确 来源尤为重要, 有助于厘清食物网获取老碳的具体途径和方式. 尽管利用混合同位素模型能够区分不同碳 源对消费者的贡献, 然而需要更为有效的方法来阐明有机碳一消费者、无机碳一消费者、有机碳一无机碳之 间的相互转化途径和过程, 现阶段已有研究尝试通过单体同位素 (compound-specific) ${ }^{14} \mathrm{C}$ 分析法追踪碳源在 食物网的详细路径.

2) 虽然研究证实老碳对水生态系统食物网的贡献, 然而现有的成果大多集中在冰冻圈地区, 老碳对食 物网的补贴现象在其他区域水体中 (温带、热带) 是否普遍存在, 现阶段开展的工作并不多, 还需要继续探索 和延伸. 此外, 消费者摄食老碳的控制因素还不明确, 因此, 亟需研究水体中老碳的分布形态、组成成分、物 理化学性质以及生物可利用性, 结合室内和自然条件下的原位试验, 揭示老碳参与食物网碳循环的内在机 理和影响因素.

3) 老碳对食物网的补贴一定程度上缓解了水生态系统自身初级生产的不足, 在一些水体中甚至取代初 级生产者的功能和作用, 尤其是在高纬度、高海拔的冰冻圈地区. 冰冻圈水体营养水平普遍偏低、初级生产 不足, 夏季接受冰川、冻土输送的大量生物可利用性高的老碳; 而在冬季, 由于冰川融水减少, 冻融作用微 弱, 经由冰川、冻土输送的老碳数量急剧下降, 碳源剧烈的季节性变化对冰冻圈水生态系统有何具体影响尚 不清楚. 因此, 相比较无老碳贡献的系统, 由老碳引起的食物网碳源的变化是否改变了水生态系统群落结 构、生态系统功能以及系统内部的生物地球化学循环过程,都需要进一步探索.

\section{7 参考文献}

[ 1 ] Carpenter SR, Cole JJ, Pace ML et al. Ecosystem subsidies: terrestrial support of aquatic food webs from ${ }^{13} \mathrm{C}$ addition to contrasting lakes. Ecology, 2005, 86: 2737-2750.

[ 2 ] Cole JJ, Carpenter SR, Pace ML et al. Differential support of lake food webs by three types of terrestrial organic carbon. Ecology Letters, 2006, 9: 558-568.

[ 3 ] Pace ML, Carpenter SR, Cole JJ et al. Does terrestrial organic carbon subsidize the planktonic food web in a clear-water lake? Limnology and Oceanography, 2007, 52: 2177-2189.

[ 4 ] Fontaine S, Barot S, Barré P et al. Stability of organic carbon in deep soil layers controlled by fresh carbon supply. Nature, 2007, 450: 277-280.

[ 5 ] Guenet B, Danger M, Abbadie L et al. Priming effect: bridging the gap between terrestrial and aquatic ecology. Ecology, 
2010, 91 : 2850-2861.

[ 6 ] Bianchi TS. The role of terrestrially derived organic carbon in the coastal ocean: A changing paradigm and the priming effect. Proceedings of the National Academy of Sciences, 2011, 108: 19473-19481.

[ 7 ] Ekschmitt K, Liu M, Vetter S et al. Strategies used by soil biota to overcome soil organic matter stability-why is dead organic matter left over in the soil? Geoderma, 2005, 128: 167-176.

[ 8 ] Heimann M, Reichstein M. Terrestrial ecosystem carbon dynamics and climate feedbacks. Nature, 2008, 451: $289-292$.

[ 9 ] Trumbore S. Radiocarbon and soil carbon dynamics. Annual Review of Earth and Planetary Sciences, 2009, 37: 47-66.

[10] Gurwick NP, McCorkle DM, Groffman PM et al. Mineralization of ancient carbon in the subsurface of riparian forests. Journal of Geophysical Research: Biogeosciences, 2015, 113(G2) : 143-144.

[11] McCallister SL, del Giorgio PA. Evidence for the respiration of ancient terrestrial organic C in northern temperate lakes and streams. Proceedings of the National Academy of Sciences, 2012, 109: 16963-16968.

[12] Raymond PA, Bauer JE, Caraco NF et al. Controls on the variability of organic matter and dissolved inorganic carbon ages in northeast US rivers. Marine Chemistry, 2004, 92: 353-366.

[13] Guo L, Ping CL, Macdonald RW. Mobilization pathways of organic carbon from permafrost to arctic rivers in a changing climate. Geophysical Research Letters, 2007, 34(13) : 173-180.

[14] Hood E, Fellman J, Spencer RGM et al. Glaciers as a source of ancient and labile organic matter to the marine environment. Nature, 2009, 462: 1044-1047.

[15] Singer GA, Fasching C, Wilhelm L et al. Biogeochemically diverse organic matter in Alpine glaciers and its downstream fate. Nature Geoscience, 2012, 5: 710-714.

[16] Bhatia MP, Das SB, Xu L et al. Organic carbon export from the Greenland ice sheet. Geochimica et Cosmochimica Acta, 2013, 109: 329-344.

[17] Spencer RGM, Guo W, Raymond PA et al. Source and biolability of ancient dissolved organic matter in glacier and lake ecosystems on the Tibetan Plateau. Geochimica et Cosmochimica Acta, 2014, 142 : 64-74.

[18] Mann PJ, Eglinton TI, McIntyre CP et al. Utilization of ancient permafrost carbon in headwaters of Arctic fluvial networks. Nature communications, 2015, 6: 7856.

[19] Fernandes R, Rinne C, Nadeau MJ et al. Towards the use of radiocarbon as a dietary proxy : establishing a first wide-ranging radiocarbon reservoir effects baseline for Germany. Environmental Archaeology, 2016, 21 : 285-294.

[20] Fellman JB, Hood E, Raymond PA et al. Evidence for the assimilation of ancient glacier organic carbon in a proglacial stream food web. Limnology and Oceanography, 2015, 60: 1118-1128.

[21] Hågvar S, Ohlson M, Brittain JE. A melting glacier feeds aquatic and terrestrial invertebrates with ancient carbon and supports early succession. Arctic, Antarctic, and Alpine Research, 2016, 48: 551-562.

[22] Hu E, He H, Su Y et al. Use of multi-carbon sources by zooplankton in an oligotrophic lake in the Tibetan Plateau. Water, 2016, 8: 565 .

[23] Bellamy AR, Bauer JE, Grottoli AG. Influence of land use and lithology on sources and ages of nutritional resources for stream macroinvertebrates: a multi-isotopic approach. Aquatic Sciences, 2017, 79: 925-939.

[24] Schell DM. Carbon-13 and carbon-14 abundances in Alaskan aquatic organisms: delayed production from peat in arctic food webs. Science, 1983, 219: 1068-1071.

[25] Cherrier J, Bauer JE, Druffel ERM et al. Radiocarbon in marine bacteria: Evidence for the ages of assimilated carbon. Limnology and Oceanography, 1999, 44: 730-736.

[26] McCallister SL, Bauer JE, Cherrier JE et al. Assessing sources and ages of organic matter supporting river and estuarine bacterial production: a multiple isotope $\left(\Delta^{14} \mathrm{C}, \delta^{13} \mathrm{C}\right.$, and $\left.\delta^{15} \mathrm{~N}\right)$ approach. Limnology and Oceanography, 2004, 49: 1687-1702.

[27] Caraco N, Bauer JE, Cole JJ et al. Millennial-aged organic carbon subsidies to a modern river food web. Ecology, 2010, 91: 2385-2393.

[28] Keaveney E, Reimer P, Foy R. Young, old, and weathered carbon-Part 1: Using radiocarbon and stable isotopes to identify carbon sources in an alkaline, Humic Lake. Radiocarbon, 2015, 57: 425-438.

[29] Ishikawa NF, Uchida M, Shibata Y et al. A new application of radiocarbon $\left({ }^{14} \mathrm{C}\right)$ concentrations to stream food web analysis. Nuclear Instruments and Methods in Physics Research Section B: Beam Interactions with Materials and Atoms, 2010, 
268: $1175-1178$.

[30] Ishikawa NF, Uchida M, Shibata Y et al. Natural C-14 provides new data for stream food-web studies: a comparison with C-13 in multiple stream habitats. Marine and Freshwater Research, 2012, 63: 210-217.

[31] Ishikawa NF, Uchida M, Shibata Y et al. Carbon storage reservoirs in watersheds support stream food webs via periphyton production. Ecology, 2014, 95: 1264-1271.

[32] Zigah PK, Minor EC, Werne JP et al. An isotopic $\left(\Delta^{14} \mathrm{C}, \delta^{13} \mathrm{C}\right.$, and $\left.\delta^{15} \mathrm{~N}\right)$ investigation of the composition of particulate organic matter and zooplankton food sources in Lake Superior and across a size-gradient of aquatic systems. Biogeosciences, 2012, 9: 3663-3678.

[33] Guillemette F, Bianchi TS, Spencer RGM. Old before your time: Ancient carbon incorporation in contemporary aquatic foodwebs. Limnology and Oceanography, 2017, 62(4) : 1682-1700.

[34] Bellamy AR, Bauer JE. Nutritional support of inland aquatic food webs by aged carbon and organic matter. Limnology and Oceanography Letters, 2017, 2 : 131-149.

[35] Cole JJ, Carpenter SR, Kitchell J et al. Strong evidence for terrestrial support of zooplankton in small lakes based on stable isotopes of carbon, nitrogen, and hydrogen. Proceedings of the National Academy of Sciences, 2011, 108: 1975-1980.

[36] Brett MT, Kainz MJ, Taipale SJ et al. Phytoplankton, not allochthonous carbon, sustains herbivorous zooplankton production. Proceedings of the National Academy of Sciences, 2009, 106: 21197-21201.

[37] Jones R. The influence of humic substances on lacustrine planktonic food chains. Hydrobiologia, 1992, 229: 73-91.

[38] Hessen D, Nygaard K. Bacterial transfer of methane and detritus: implications for the pelagic carbon budget and gaseous release. Archiv fur Hydrobiology, 1992, 37: 139-148.

[39] Berggren M, StromL, Laudon H. Lake secondary production fueled by rapid transfer of low molecular weight organic carbon from terrestrial sources to aquatic consumers. Ecology Letters, 2010, 13: 870-880.

[40] Clitherow LR, Carrivick JL, Brown LE. Food web structure in a harsh glacier-fed river. PLoS One, 2013, 8: e60899.

[41] Qin DH, Dong WJ, Luo Y eds. Climate and environment change in China: 2012 Part 1 Scientific Basis. Beijing: China Meteorological Press, 2012.

[42] Hood E, Battin TJ, Fellman J et al. Storage and release of organic carbon from glaciers and ice sheets. Nature Geoscience, 2015, 8: 91-96.

[43] Waelbroeck C, Monfray P, Oechel WC et al. The impact of permafrost thawing on the carbon dynamics of tundra. Geophysical Research Letters, 1997, 24: 229-232.

[44] Oechel WC, Vourlitis GL, Hastings SJ et al. Acclimation of ecosystem $\mathrm{CO}_{2}$ exchange in the Alaskan Arctic in response to decadal climate warming. Nature, 2000, 406: 978-981.

[45] Xu B, Cao J, Hansen J et al. Black soot and the survival of Tibetan glaciers. Proceedings of the National Academy of Sciences, 2009, 106: 22114-22118.

[46] Yao T, Thompson L, Yang W et al. Different glacier status with atmospheric circulations in Tibetan Plateau and surroundings. Nature Climate Change, 2012, 2: 663-667.

[47] Frey KE, Smith LC. Amplified carbon release from vast West Siberian peatlands by 2100. Geophysical Research Letters, 2005, 32: L09401. DOI: 10.1029/2004GL022025.

[48 ] Tittel J, Büttner O, Freier K et al. The age of terrestrial carbon export and rainfall intensity in a temperate river headwater system. Biogeochemistry, 2013, 115: 53-63.

[49] Tittel J, Müller C, Schultze M et al. Fluvial radiocarbon and its temporal variability during contrasting hydrological conditions. Biogeochemistry, 2015, 126: 57-69.

[50] Freeman C, Evans CD, Monteith DT et al. Export of organic carbon from peat soils. Nature, 2001, 412 : 785-785.

[51] Butman DE, Wilson HF, Barnes RT et al. Increased mobilization of aged carbon to rivers by human disturbance. Nature Geoscience, 2015, 8: 112-116.

[52] Schuur EAG, Vogel JG, Crummer KG et al. The effect of permafrost thaw on old carbon release and net carbon exchange from tundra. Nature, 2009, 459: 556-559.

[53] Aiken GR, Spencer RGM, Striegl RG et al. Influences of glacier melt and permafrost thaw on the age of dissolved organic carbon in the Yukon River basin. Global Biogeochemical Cycles, 2014, 28: 525-537.

[54] Guo L, Semiletov I, Gustafsson Ö et al. Characterization of Siberian Arctic coastal sediments: Implications for terrestrial 
organic carbon export. Global Biogeochemical Cycles, 2004, 18: GB1036. DOI: 10.1029/2003GB002087.

[55] Guo L, Macdonald RW. Source and transport of terrigenous organic matter in the upper Yukon River: evidence from isotope $\left(\delta^{13} \mathrm{C}, \Delta^{14} \mathrm{C}\right.$, and $\left.\delta^{15} \mathrm{~N}\right)$ composition of dissolved, colloidal, and particulate phases. Global Biogeochemical Cycles, 2006, 20(2): GB2001. DOI: 10.1029/2005GB002593.

[56] Hedges JI, Oades JM. Comparative organic geochemistries of soils and marine sediments. Organic geochemistry, 1997, 27: 319-361.

[57] Post DM. Using stable isotopes to estimate trophic position: models, methods, and assumptions. Ecology, 2002, 83: 703-718

[58] Hamilton SK, Sippel SJ, Bunn SE. Separation of algae from detritus for stable isotope or ecological stoichiometry studies using density fractionation in colloidal silica. Limnology and Oceanography: Methods, 2005, 3: 149-157.

[59] Middelburg JJ. Stable isotopes dissect aquatic food webs from the top to the bottom. Biogeosciences, 2014, 11: $2357-2371$.

[60] Petsch ST, Eglinton TI, Edwards KJ. 14C-dead living biomass: evidence for microbial assimilation of ancient organic carbon during shale weathering. Science, 2001, 292: 1127-1131.

[61] Zigah PK, Minor EC, Werne JP et al. Radiocarbon and stable carbon isotopic insights into provenance and cycling of carbon in Lake Superior. Limnology and Oceanography, 2011, 56: 867-886.

[62] Stuiver M. Workshop on ${ }^{14} \mathrm{C}$ data reporting. Radiocarbon, 1980, 22 : 964-966.

[63] Jansson M. Allochthonous organic carbon decreases pelagic energy mobilization in lakes. Limnology and Oceanography, 2003, 48: 1711-1716.

[64] Kelly PT, Solomon CT, Weidel BC et al. Terrestrial carbon is a resource, but not a subsidy, for lake zooplankton. Ecology, 2014, 95 : 1236-1242.

[65] Guillemette F, McCallister SL, del Giorgio PA. Selective consumption and metabolic allocation of terrestrial and algal carbon determine allochthony in lake bacteria. The ISME journal, 2016, 10: 1373-1382.

[66] Cottingham KL. Nutrients and zooplankton as multiple stressors of phytoplankton communities: evidence from size structure. Limnology and Oceanography, 1999, 44: 810-827.

[67] Karlsson J, Berggren M, Ask J et al. Terrestrial organic matter support of lake food webs: evidence from lake metabolism and stable hydrogen isotopes of consumers. Limnology and oceanography, 2012, 57: 1042-1048.

[68] Wilkinson GM, Carpenter SR, Cole JJ et al. Terrestrial support of pelagic consumers: patterns and variability revealed by a multi-lake study. Freshwater Biology, 2013, 58: 2037-2049.

[69] Aberg J, Jansson M, Karlsson J. Pelagic and benthic net production of dissolved inorganic carbon in an unproductive subarctic lake. Freshwater Biology, 2007, 52 : 549-560.

[70 C Cooke SL, Fischer JM, Kessler K et al. Direct and indirect effects of additions of chromophoric dissolved organic matter on zooplankton during large scale mesocosm experiments in an oligotrophic lake. Freshwater Biology, 2015, 60: 2362-2378.

[71 ] Yoneda M, Hirota M, Uchida M et al. Marine radiocarbon reservoir effect in the western North Pacific observed in archaeological fauna. Radiocarbon, 2001, 43: 465-472.

[72] Dutta K, Bhushan R, Somayajulu BK. Delta R correction values for the northern Indian Ocean.Radiocarbon, 2001,43 : 483-488.

[73] Deevey JrES, Gross MS, Hutchinson GE et al. The natural C14 contents of materials from hard-water lakes. Proceedings of the National Academy of Sciences of the United States of America, 1954, 40: 285.

[74] Cook GT, Bonsall C, Hedges REM et al. A freshwater diet-derived C-14 reservoir effect at the stone age sites in the iron gates gorge. Radiocarbon, 2001, 43: 453-460. 